\title{
Large directed flow of open charm mesons probes the three dimensional distribution of matter in heavy ion collisions
}

\author{
Sandeep Chatterję $\&^{*}$ and Piotr Bożel \\ AGH University of Science and Technology, \\ Faculty of Physics and Applied Computer Science, \\ aleja Mickiewicza 30, 30-059 Krakow, Poland
}

Abstract

Thermalized matter created in non-central relativistic heavy-ion collisions is expected to be tilted in the reaction plane with respect to the beam axis. The most notable consequence of this forward-backward symmetry breaking is the observation of rapidity-odd directed flow for charged particles. On the other hand, the production points for heavy quarks are forward-backward symmetric and shifted in the transverse plane with respect to the fireball. The drag on heavy quarks from the asymmetrically distributed thermalized matter generates substantial directed flow for heavy flavor mesons. We predict a very large rapidity odd directed flow of $D$ mesons in non-central $\mathrm{Au}$-Au collisions at $\sqrt{s_{N N}}=200 \mathrm{GeV}$, several times larger than for charged particles. A possible experimental observation of a large directed flow for heavy flavor mesons would represent an almost direct probe of the 3-dimensional distribution of matter in heavy-ion collisions.

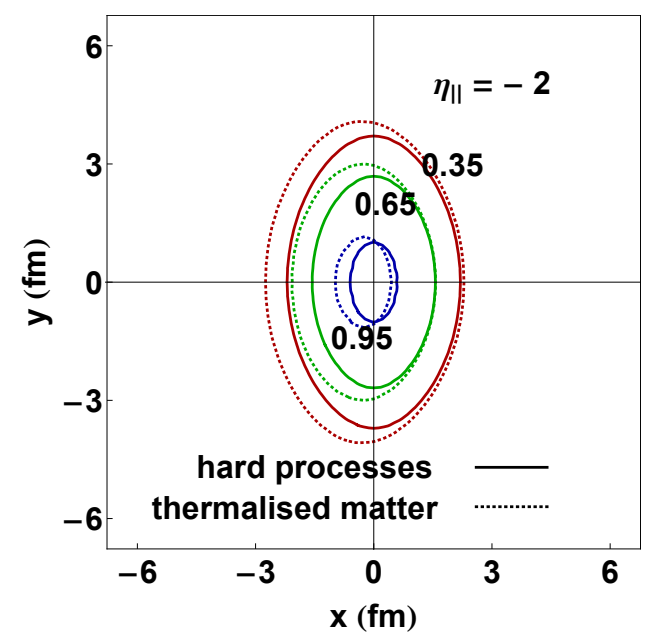

FIG. 1. The transverse density profile of the fireball tilted relative to the beam axis and the binary collision profile at $\eta_{\|}=-2$.

The dynamics of relativistic heavy-ion collisions can be largely factorized into a part that is transverse to the beam direction and the longitudinal dynamics that is parallel to it. The transverse evolution has been extensively investigated with ample evidence of collective expansion [1. The longitudinal dynamics is usually studied in two aspects, event by event fluctuations of the longitudinal distribution of matter [2] and the breaking of the beam axis symmetry in non-central collisions [3, 4]. One of the key question towards understanding the longitudinal evolution is how the forward-backward symmetry is broken in the initial state for symmetric but non-central

* Sandeep.Chatterjee@fis.agh.edu.pl

$\dagger$ Piotr.Bozek@fis.agh.edu.pl collision systems. The determination of the strength and mechanism of this symmetry breaking will lead to a significant progress of our understanding of energy deposition in relativistic collisions.

At intermediate beam energies, charged particle directed flow $v_{1}$ is sensitive to the QCD equation of state [5]. At higer energies $\left(\sqrt{s_{N N}}=200 \mathrm{GeV}\right.$ and above), it is a key observable that probes the longitudinal profile of the fireball [4]. Heavy quarks with masses that are several times larger than the fireball temperature are mostly produced in the initial stages of the collision. Hence they witness the entire space-time evolution of the fireball and are ideal candidates to probe initial state physics [6]. We demonstrate the possibility of probing the initial state longitudinal profile of the thermalized matter through the directed flow of heavy quarks.

A natural suggestion to explain the rapidity spectra of produced particles in asymmetric collisions like $\mathrm{d}+\mathrm{Au}$ [7] is that each participant source preferably showers particles along its direction of motion [2, 8,. Such a picture is also well motivated by the parton model [9] as well as saturation models of QCD [10]. In order to obtain such a final state within a hydrodynamic framework, the following ansatz for the initial entropy density $s\left(\tau_{0}, x, y, \eta_{\|}\right)$ within a two-component Glauber model has been proposed 4]

$$
\begin{aligned}
s\left(\tau_{0}, x, y, \eta_{\|}\right)= & s_{0}\left[\alpha N_{\text {coll }}+(1-\alpha)\left(N_{\text {part }}^{+} f_{+}\left(\eta_{\|}\right)+\right.\right. \\
& \left.\left.N_{\text {part }}^{-} f_{-}\left(\eta_{\|}\right)\right)\right] f\left(\eta_{\|}\right)
\end{aligned}
$$

where $N_{\text {coll }}, N_{\text {part }}^{+}$and $N_{\text {part }}^{-}$are the densities for number of binary collisions and participants from the forward and backward going nuclei respectively evaluated at $(x, y)$. $\tau=\sqrt{t^{2}-z^{2}}$ and $\eta_{\|}=\frac{1}{2} \log \frac{(t+z)}{(t-z)}$ are the proper time and spacetime rapidities respectively. $\tau_{0}$ is the initial 
proper time to start hydrodynamic evolution.

$$
f\left(\eta_{\|}\right)=\exp \left(-\theta\left(\left|\eta_{\|}\right|-\eta_{\|}^{0}\right) \frac{\left(\left|\eta_{\|}\right|-\eta_{\| \mid}^{0}\right)^{2}}{2 \sigma^{2}}\right)
$$

is the longitudinal profile (for $\left|\eta_{\|}\right|<y_{\text {beam }}$ ) taken to reproduce the final state rapidity spectra of charged particles. $\alpha$ controls the admixture of the participant and binary sources contribution to the total entropy deposition for the fireball. For a good description of the $\mathrm{Au}+\mathrm{Au}$ data at $\sqrt{s_{\mathrm{NN}}}=200 \mathrm{GeV}$, we set $\eta_{\|}^{0}=1.3, \sigma=1.5$ and $\alpha=0.15$.

$f_{+,-}\left(\eta_{\|}\right)$are the profiles which introduce the rapidity odd component to the initial state

$$
f_{+}\left(\eta_{\|}\right)=\left\{\begin{array}{lr}
0, & \eta_{\|}<-\eta_{T} \\
\frac{\eta_{T}+\eta_{\|}}{2 \eta_{T}}, & -\eta_{T} \leq \eta_{\|} \leq \eta_{T} \\
1, & \eta_{\|}>\eta_{T}
\end{array}\right.
$$

with $f_{-}\left(\eta_{||}\right)=f_{+}\left(-\eta_{||}\right)$. The above tilted initial condition has been shown to describe the rapidity odd $v_{1}$ of charged particles 4 measured by STAR 11 and PHOBOS [12]. The magnitude of the tilt of the initial source is determined by the parameter $\eta_{T}$. Typically we use $\eta_{T} \simeq y_{\text {beam }}-2=3.36$, the value extracted from $\mathrm{d}+\mathrm{Au}$ collisions [8, but to demonstrate the great sensitivity of heavy flavor flow to initial tilt we will also vary $\eta_{T}$ between 2 and the beam rapidity $y_{\text {beam }}=5.36$.

The transverse profile of the entropy density for the fireball and of the binary collision sources at $\eta_{\|}=-2$ for $\mathrm{Au}-\mathrm{Au}$ collision at impact parameter $b=8.3 \mathrm{fm}$ is plotted in Fig. 1. The Au nucleus flying with the positive (negative) rapidity is located at $x=b / 2(-b / 2)$. The fireball is tilted to positive (negative) $x$ with respect to the beam axis for positive (negative) $\eta_{\|}$. The tilted fireball develops a rapidity-odd directed flow $v_{1}$ of charged particles in the hydrodynamic expansion [4, 13, consistent with experimental data [1].

Charm quarks produced by hard binary collisions between nulceons from the two colliding nuclei are forwardbackward symmetric in rapidity. The distribution of the production points of charm quarks is according to the binary collision profile which is symmetric with respect to the beam axis. However, the shift in the bulk and binary collision profiles means that heavy quarks are produced at points in the transverse plane shifted with respect to the center of the fireball. This results in the enhanced dipole asymmetry in the heavy quark flow pattern. There is a possibility for the heavy quark profile to develop a tiny tilt due to correction from nucleon position dependent nuclear modifications of parton distribution functions. In this first study we ignore such effects.

The matter in the fireball is evolved with $3+1$ dimensional relativistic viscous hydrodynamics using the HLLE algorithm [14]. We use a shear viscosity $\eta / s=0.08$ and bulk viscosity $\zeta / s=0.04$ in the hadronic phase. A nonzero bulk viscosity in the hadronic phase mimics the less effective equilibration processes. We use a cross-over equation of state, interpolating between lattice QCD data and hadron resonance gas model [15. Note that the discussed mechanism of symmetry breaking does not require event by event fluctuations and all calculations are performed using smooth initial conditions (Eq. 1) from the nucleon Glauber model. A rapidity-odd directed flow of charged particles of similar magnitude has been predicted in event-by-event simulations as well [15].

The hydrodynamic evolution provides us the spacetime history of the fluid velocity $u^{\mu}$ and temperature $T$. We study the phase space evolution of heavy flavor, particularly charm quarks in this background through Langevin dynamics

$$
\begin{aligned}
\Delta \mathbf{r}_{i} & =\frac{\mathbf{p}_{i}}{E} \Delta t \\
\Delta \mathbf{p}_{i} & =-\gamma \mathbf{p}_{i} \Delta t+\rho_{i} \sqrt{2 D \Delta t}
\end{aligned}
$$

where $\Delta \mathbf{r}$ and $\Delta \mathbf{p}$ refer to the updates of the position and momentum vectors of the heavy quark respectively in time $\Delta t . i=x, y$ and $z$ refers to the three components in Cartesian coordinates. $\gamma$ and $D$ are the drag and diffusion coefficients respectively that characterize the interaction between the heavy quark and the medium. We have assumed a diagonal form for the diffusion matrix here which is often employed in the study of heavy quark dynamics related to heavy ion phenomenology [16 18]. $\rho_{i}$ is randomly sampled from a normal distribution at every time step such that $\left\langle\rho_{i}\right\rangle=0$ and $\left\langle\rho_{i} \rho_{j}\right\rangle=\delta_{i j}$. We work in the post-point realization of this stochastic term and take $D=\gamma E T$, where $E=\sqrt{p^{2}+m^{2}}$ is the energy of the heavy quark with mass $m$. This ensures the long time approach of the heavy quark phase space distribution to the equilibrium Boltzmann-Juttner distribution [19].

The initial phase space distribution of the heavy quarks is sampled from the binary collision profile and from the $p_{T}$ spectra obtained in $\mathrm{p}+\mathrm{p}$ collisions [25]. The Langevin dynamics as written in Eqs. 4 and 5 holds true in a static medium. Thus, we perform Lorentz transformation on the heavy quark phase space coordinates to obtain the corresponding coordinates in the local rest frame of the fluid following which the position and momentum are updated according to the Langevin evolution of Eqs. 4 and 5. Finally, we perform inverse Lorentz transformation to obtain the heavy quark phase space coordinates in the laboratory rest frame. At the end of the Langevin evolution (when the temperature drops to $150 \mathrm{MeV}$ ), we hadronize the charm quarks via Petersen fragmentation [26] to obtain the final momentum distribution of the $D$ and $\bar{D}$ mesons.

The experimental observables for heavy flavor mesons 

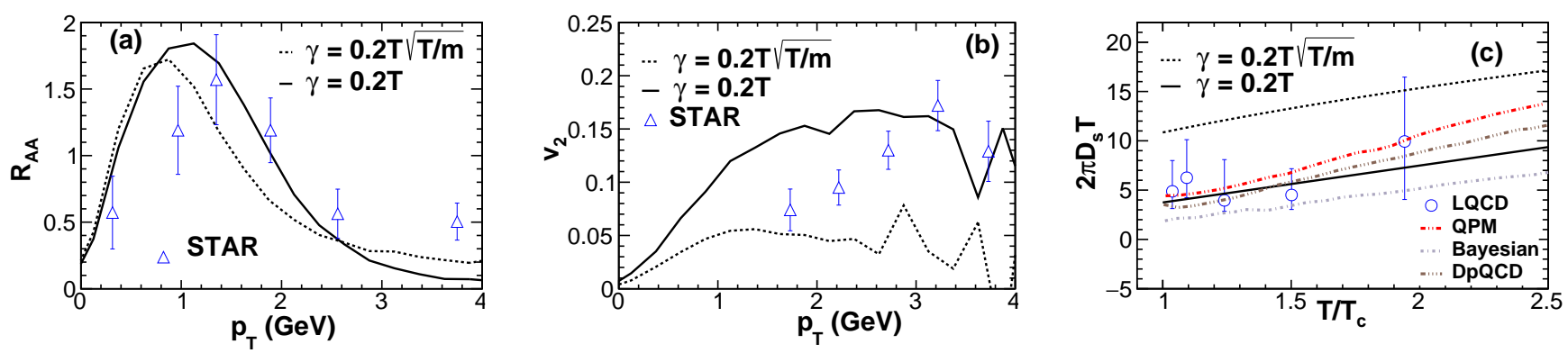

FIG. 2. (a) Nuclear modification factor for D mesons at mid-rapidity as measured in $(0-10) \%$ centrality Au+Au at $\sqrt{s_{\mathrm{NN}}}=200 \mathrm{GeV}$ [20] compared to our calculation for two different choices for the temperature dependence of the drag coefficient, $\gamma=0.2 T$ and $\gamma=0.2 T \sqrt{T / m}$. (b) Elliptic flow at mid-rapidity as measured in $(0-80) \%$ centrality Au+Au at $\sqrt{s_{\mathrm{NN}}}=200 \mathrm{GeV}$ 21] compared to our calculation for the two different choices of $\gamma$. (c) The spatial diffusion constant for the two choices of $\gamma$ in this work compared to results from lattice calculations 22, quasiparticle models [18, 23] and Bayesian analysis of the data 24 .
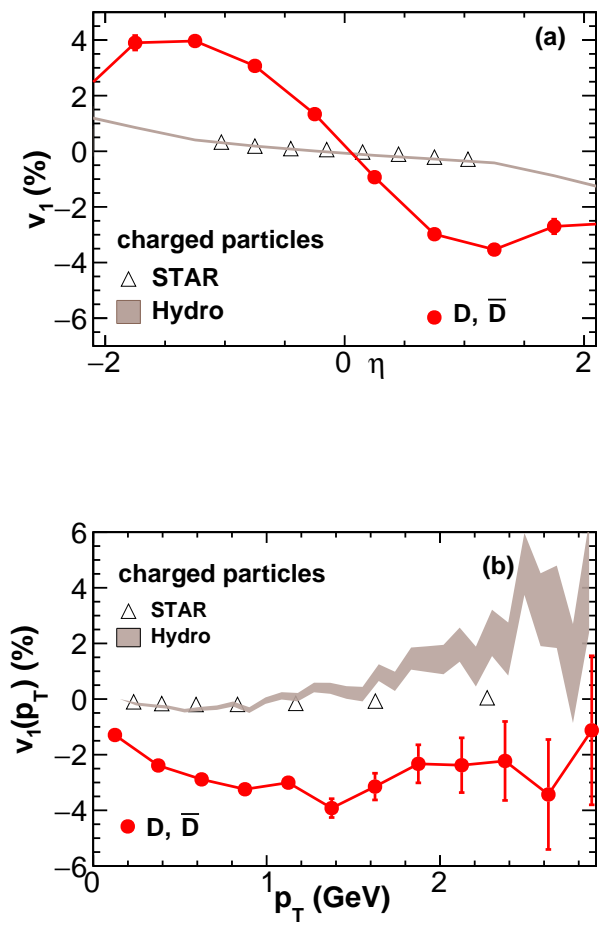

FIG. 3. (a) Rapidity dependence of $v_{1}$ for $\mathrm{D}$ mesons as obtained for $\eta_{T}=3.36$ and $\gamma=0.2 T$ (filled circles). The STAR measurements [11] (open triangles), as well as our results from the hydrodynamic model (shaded band) for charged particle $v_{1}$ is also shown for comparison. (b) Same as (a) for $p_{T}$ differential $v_{1}$.

are the nuclear suppression factor

$$
R_{A A}=\frac{d N^{A A} / d p_{T}}{N_{\text {coll }} d N^{p p} / d p_{T}},
$$

defined as the scaled ratio of $p_{T}$ spectra in $\mathrm{Au}+\mathrm{Au}$ and $\mathrm{p}+\mathrm{p}$ collisions, and the directed $v_{1}$ and elliptic $v_{2}$ harmonic flow coefficients of the azimuthal angle distribution for $D$ mesons

$$
\frac{d N}{d \phi} \propto 1+2 v_{1} \cos \left(\phi-\Psi_{1}\right)+2 v_{2} \cos \left(2\left(\phi-\Psi_{2}\right)\right)+\ldots .
$$

The elliptic flow is defined with respect to the second order event plane direction $\Psi_{2}$ for charged particles and the rapidity odd directed flow is defined with respect to the reaction plane $\Psi_{1}$. In the simulation the reaction plane is well defined, in the experiment it is usually defined from the spectators (e.g. see 5] for a discussion).

The jury is still out on the determination of the drag experienced by the charm quark in the medium [18, 23. 24, 27. 29]. We work with a simple ansatz,

$$
\gamma=\gamma_{0} T(T / m)^{x}
$$

and adjust $\gamma_{0}$ and $x$ for a qualitative description of the measured values of $R_{A A}\left(p_{T}\right)$ [20] and $v_{2}\left(p_{T}\right)$ [21] at midrapidity. The results are shown in Fig. 2. A good qualitative description of the data is found for $\gamma_{0}=0.2$ and $x=0-0.5$. A different choice, $\gamma=\gamma_{0} T(T / E)^{x}$ does not lead to further improvements. In Fig. 2 (c), we have compare the $T$ dependence of the dimensionless quantity $2 \pi D_{s} T\left(D_{s}\right.$ is the charm quark diffusion coefficient in the coordinate space) to results from lattice calculations 22, quasiparticle models [18, 23, and Bayesian analysis of the data 24, lattice data slightly favors the choice $\gamma \propto T$ for the ansatz in Eq. 8. Nevertheless, we study also the case $\gamma \propto T^{1.5}$ for completeness. Different choices do not modify our conclusions qualitatively.

To make a useful prediction for $v_{1}$ for experiments with limited statistics we show the $0-80 \%$ centrality results. This corresponds to a choice of the impact parameter $b=8.3 \mathrm{fm}$ in the Glauber model to generate the initial conditions. The results shown in Fig. 3 correspond to $\gamma \propto$ $T$. Charged particle spectra are calculated in a statistical 
emission model and resonance decays at the freeze-out temperature $150 \mathrm{MeV}$ [30]. The agreement of the directed flow coefficient $v_{1}$ for charged particles with experiment [4] is reproduced in our calculation.

In Fig. 3 (a), we show the results for the $p_{T}$ integrated $v_{1}$ in different $\eta$ bins. The most notable observation is that for central rapidities the predicted directed flow coefficient for heavy flavors is several times larger than the $v_{1}$ of charged particles measured by STAR. It is a very strong, distinctive signal of symmetry breaking in the initial conditions. Hydrodynamic expansion of the tilted source results in a moderate value of the odd-component of the directed flow for charged particles stemming from the thermalized fireball. The heavy quark production points are shifted in the transverse plane with respect to the bulk of the matter. The interaction of the medium in the fireball on heavy quarks is biased resulting in a larger directed flow of heavy flavor mesons. We note that our prediction for heavy flavor $v_{1}$ at central rapidities is approximately three times larger than in the results of earlier calculations using a transport model [31].

Initially, the bulk of matter is tilted which translates into a tilted temperature profile. This sets off the hydrodynamic response and the directed flow of the fluid is built after some time [4]. Thus, there could be two factors that drive the large $v_{1}$ of heavy flavors, preferential scattering of the heavy quarks by the tilted source and secondly, the drag by the directed flow of the bulk matter. We have explicitly investigated this by using boost invariant $T$ or $u^{\mu}$ and found that the large heavy flavor $v_{1}$ is mostly due to the drag of the fluid.

In Fig. $3(\mathrm{~b})$, we show the $p_{T}$ differential $\operatorname{sgn}(\eta) v_{1}$ in the $|\eta| \leq 1.3$ range. The $p_{T}$ dependence of $v_{1}$ is similar to that of $v_{2}$ from Fig. 2 (b): it rises with $p_{T}$ for $p_{T}<1$ $\mathrm{GeV}$ and then stays constant up to $p_{T} \sim 2 \mathrm{GeV}$ before slowly falling off. The $p_{T}$ range where the directed flow magnitude is large is also the $p_{T}$ range where $R_{A A}>1$ as seen in Fig. 2 (a). The relative modification of the $p_{T}$ for heavy quarks in this momentum range due to a drag from the collective motion of matter is the largest, and it is at the origin of both the increase of $R_{A A}$ and a large $v_{1}$. At higher $p_{T}$, jet quenching by the tilted fireball [10] could be a source of additional $v_{1}$ besides the drag by the tilted bulk matter that we discuss. The spectra of heavy flavor mesons are shifted towards negative $p_{x}$. The bias from the off-center position of the fireball (Fig. 1) leads to a net transfer of $p_{x}$ to heavy quarks.

The observation of enhanced forward-backward dipole asymmetry in the flow of heavy quarks is best depicted by the $v_{1}$ slope at mid-rapidity. This is shown in Fig. 4 . The slope has been extracted by fitting to the $v_{1}$ in the $|\eta|<1$ range. Depending on the choice of the parameters the slope for the D mesons is $5-20$ times larger than that of the charged particles. A notable feature of the directed flow of heavy flavors is its sensitivity to the magnitude of

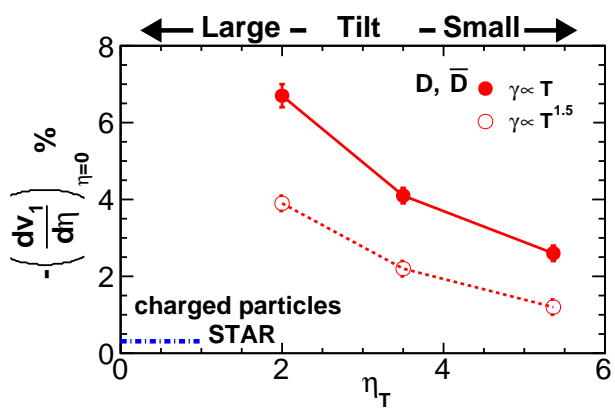

FIG. 4. Slope of the directed flow at mid-rapidity for D and $\bar{D}$ mesons compared to that of the STAR measurement of mid-rapidity slope for charged particles [1].

the initial tilt of the fireball. By varying $\eta_{T}$ governing the asymmetry of the initial entropy deposition of the left and right going sources (Eq. 3) the initial tilt can be varied. When changing $\eta_{T}$ from $y_{\text {beam }}$ (small tilt) to $0.4 y_{\text {beam }}$ (large tilt), the change in charged particle $v_{1}$ is moderate, $\sim 0.5 \%$ at $\eta=0.75$. On the other hand, the heavy flavor $v_{1}$ at the same $\eta$ bin changes by $2-3 \%$ while the $v_{1}$ slope increases by a factor 3 . Thus, a measurement of the $v_{1}$ slope for heavy flavors would present a sensitive probe of the initial matter distribution in the transverse and longitudinal directions.

Several comments are in order. The dependence of $\gamma$ on the medium properties like $T$ and heavy quark properties like $m$ and $p$ is far from settled [18, 23, $27,[29]$. The value of the drag coefficient influences the final $v_{1}$ significantly and hence ignorance of $\gamma$ can introduce considerable uncertainty in the extraction of $\eta_{T}$. Thus, proper calibration of the medium interaction with the heavy quark and its demonstration by good simultaneous description of $p_{T}$ dependence of $R_{A A}$ and $v_{2}$ at mid-rapidity is essential. The directed flow of heavy flavors should be included in the set of observables used to constrain the drag coefficient, besides $R_{A A}$ and $v_{2}$.

We have checked that the heavy quark $v_{1}$ is robust to variations of other parameters like the initial time to start hydrodynamics $\tau_{0}$, the value of shear and bulk viscosity $\eta / s$ and $\zeta / s$. It has been suggested that hard processes in a $k_{T}$ factorized saturation framework follow a profile that is also tilted with respect to the beam axis [32]. This would reduce the heavy quark $v_{1}$ or could even reverse its sign. Thus, the relative magnitude (and sign) of heavy flavor and charged particle $v_{1}$ can distinguish between different saturation mechanism for hard processes. This emphasizes the significance of the measurement of heavy quark $v_{1}$ at different collision energies.

The strong electromagnetic fields in the initial state could contribute to heavy flavor $v_{1}$ as well [33. However, the effect of the electromagnetic fields is of opposite sign 
on $D$ and $\bar{D}$ mesons and would not influence the average directed flow of $D$ mesons.

We note that at higher energies the magnitude of the rapidity-odd directed flow of charged particles decreases. In the hydrodynamic model it results from a decrease of the initial tilt of the source. This effect would lead to a decrease of the directed flow of $D$ mesons at higher energies. On the other hand, the larger density of the fireball makes the drag of heavy quark more effective. The re- sulting $D$ meson directed flow at $\sqrt{s_{N N}}=2760 \mathrm{GeV}$ is predicted to be much larger than for charged particles.

The authors acknowledge helpful discussion with Iurii Karpenko on the vHLLE code. SC also acknowledges fruitful discussions with Jane Alam, Santosh K. Das, Vincenzo Greco and Suropriya Saha. This research is supported by the AGH UST statutory tasks No. 11.11.220.01/1 within subsidy of the Ministry of Science and Higher Educations and by the National Science Centre Grant No. 2015/17/B/ST2/00101.
[1] J.-Y. Ollitrault, J. Phys. Conf. Ser. 312, 012002 (2011)U. Heinz and R. Snellings, Ann. Rev. Nucl. Part. Sci. 63, 123 (2013)C. Gale, S. Jeon, and B. Schenke, Int. J. Mod. Phys. A28, 1340011 (2013)

[2] N. Armesto, L. McLerran, and C. Pajares, Nucl. Phys. A781, 201 (2007)A. Bzdak, Phys. Rev. C80, 024906 (2009)P. Bożek, W. Broniowski, and J. Moreira, ibid. C83, 034911 (2011)H. Petersen, V. Bhattacharya, S. A. Bass, and C. Greiner, ibid. C84, 054908 (2011)A. Bzdak and D. Teaney, ibid. C87, 024906 (2013)J. Jia and P. Huo, ibid. C90, 034905 (2014)L.-G. Pang, G.-Y. Qin, V. Roy, X.-N. Wang, and G.-L. Ma, ibid. C91, 044904 (2015)A. Monnai and B. Schenke, Phys. Lett. B752, 317 (2016)

[3] L. P. Csernai and D. Rohrich, Phys. Lett. B458, 454 (1999)R. J. M. Snellings, H. Sorge, S. A. Voloshin, F. Q. Wang, and N. Xu, Phys. Rev. Lett. 84, 2803 (2000)M. A. Lisa, U. W. Heinz, and U. A. Wiedemann, Phys. Lett. B489, 287 (2000)F. Becattini, L. P. Csernai, and D. J. Wang, Phys. Rev. C88, 034905 (2013)

[4] P. Bożek and I. Wyskiel, Phys. Rev. C81, 054902 (2010)

[5] J. Steinheimer, J. Auvinen, H. Petersen, M. Bleicher, and H. Stöcker, Phys. Rev. C89, 054913 (2014)Y. Nara, H. Niemi, J. Steinheimer, and H. Stöcker, Phys. Lett. B769, 543 (2017)

[6] R. Rapp and H. van Hees (2010) pp. 111-206, arXiv:0903.1096 [hep-ph]A. Andronic et al., Eur. Phys. J. C76, 107 (2016)G. Aarts et al., ibid. A53, 93 (2017)J. Noronha-Hostler, B. Betz, J. Noronha, and M. Gyulassy, Phys. Rev. Lett. 116, 252301 (2016)

[7] R. Nouicer et al. (PHOBOS Collaboration), J. Phys. G30, S1133 (2004)

[8] A. Bialas and W. Czyz, Acta Phys. Polon. B36, 905 (2005)

[9] S. J. Brodsky, J. F. Gunion, and J. H. Kuhn, Phys. Rev. Lett. 39, 1120 (1977)

[10] A. Adil and M. Gyulassy, Phys. Rev. C72, 034907 (2005)

[11] B. I. Abelev et al. (STAR Collaboration), Phys. Rev. Lett. 101, 252301 (2008)

[12] B. B. Back et al. (PHOBOS Collaboration), Phys. Rev. Lett. 97, $01 \overline{2301}(2006)$

[13] R. Ryblewski and W. Florkowski, Phys. Rev. C85, 064901 (2012)V. P. Konchakovski, W. Cassing, Yu. B. Ivanov, and V. D. Toneev, ibid. C90, 014903 (2014)F. Be- cattini, G. Inghirami, V. Rolando, A. Beraudo, L. Del Zanna, A. De Pace, M. Nardi, G. Pagliara, and V. Chandra, Eur. Phys. J. C75, 406 (2015)

[14] I. Karpenko, P. Huovinen, and M. Bleicher, Comput. Phys. Commun. 185, 3016 (2014)

[15] P. Bożek, Phys. Rev. C85, 034901 (2012)

[16] H. van Hees, V. Greco, and R. Rapp, Phys. Rev. C73, 034913 (2006)

[17] S. Cao and S. A. Bass, Phys. Rev. C84, 064902 (2011)

[18] F. Scardina, S. K. Das, V. Minissale, S. Plumari, and V. Greco, Phys. Rev. C96, 044905 (2017)

[19] M. He, H. van Hees, P. B. Gossiaux, R. J. Fries, and R. Rapp, Phys. Rev. E88, 032138 (2013)

[20] L. Adamczyk et al. (STAR Collaboration), Phys. Rev. Lett. 113, $142 \overline{301(2014)}$

[21] L. Adamczyk et al. (STAR Collaboration), Phys. Rev. Lett. 118, 212 $\overline{301(2017)}$

[22] D. Banerjee, S. Datta, R. Gavai, and P. Majumdar, Phys. Rev. D85, 014510 (2012)

[23] H. Berrehrah, P. B. Gossiaux, J. Aichelin, W. Cassing, J. M. Torres-Rincon, and E. Bratkovskaya, Phys. Rev. C90, 051901 (2014)

[24] Y. Xu, S. Cao, M. Nahrgang, J. E. Bernhard, and S. A. Bass(2017), arXiv:1710.00807 [nucl-th]

[25] M. Cacciari, P. Nason, and R. Vogt, Phys. Rev. Lett. 95, 122001 (2005)S. K. Das, F. Scardina, S. Plumari, and V. Greco, Phys. Rev. C90, 044901 (2014)

[26] C. Peterson, D. Schlatter, I. Schmitt, and P. M. Zerwas, Phys. Rev. D27, 105 (1983)S. K. Das, F. Scardina, S. Plumari, and V. Greco, Phys. Lett. B747, 260 (2015)

[27] H. van Hees and R. Rapp, Phys. Rev. C71, 034907 (2005)

[28] G. D. Moore and D. Teaney, Phys. Rev. C71, 064904 (2005)

[29] S. S. Gubser, Phys. Rev. D76, 126003 (2007)

[30] M. Chojnacki, A. Kisiel, W. Florkowski, and W. Broniowski, Comput. Phys. Commun. 183, 746 (2012)

[31] E. L. Bratkovskaya, W. Cassing, H. Stöcker, and N. Xu, Phys. Rev. C71, 044901 (2005)

[32] A. Adil, M. Gyulassy, and T. Hirano, Phys. Rev. D73, 074006 (2006)

[33] S. K. Das, S. Plumari, S. Chatterjee, J. Alam, F. Scardina, and V. Greco, Phys. Lett. B768, 260 (2017) 